\title{
TechBriefs
}

\section{FACIAL RECOGNITION}

\author{
Dr. Joshua A. Kroll, Lead Author
}

\section{PROBLEM}

Facial recognition use is increasing despite the technology's fundamental limitations, creating profound privacy and ethical challenges.

\section{POLICY IMPLICATIONS}

Facial recognition undermines privacy in new ways, including requiring individuals to avoid public spaces to opt out.

> Facial recognition is insufficiently trustworthy in many high-stakes applications to replace identification by a human.

- Errors by facial recognition systems continue to disproportionately and adversely affect women, minority, and other marginalized populations.

Claims that facial recognition technology can be used for purposes other than identifying people, such as emotion recognition, are not scientifically justifiable.

\section{FACIAL RECOGNITION: BY THE NUMBERS}

\begin{tabular}{|c|c|}
\hline 30 & $\begin{array}{l}\text { Hours in a Detroit jail spent by a Black American man falsely arrested based solely on an erroneous } \\
\text { automated facial "identification" from surveillance video. }\end{array}$ \\
\hline 11 & $\begin{array}{l}\text { Minimum number of European nations using biometric recognition systems in criminal investigations per } \\
\text { a Green Party study for the European Parliament. }{ }^{2}\end{array}$ \\
\hline 8 & Number poised in late 2021 to begin doing so. ${ }^{3}$ \\
\hline 0 & $\begin{array}{l}\text { Number of public spaces in which the European Data Protection Supervisor says the use of facial } \\
\text { recognition systems is consistent with "fundamental rights and freedoms." }\end{array}$ \\
\hline 25 & $\begin{array}{l}\text { Minimum number of US states, counties, cities, and other jurisdictions that have banned, suspended, or } \\
\text { heavily restricted use of facial recognition systems. }\end{array}$ \\
\hline 82.6 & Millions of Americans, of a total 331.5 million, who live in those jurisdictions. ${ }^{6}$ \\
\hline 10-100 & $\begin{array}{l}\text { Number of times more likely facial recognition systems are to falsely identify Black and Asian faces than } \\
\text { white faces in NIST study of } 189 \text { systems. }\end{array}$ \\
\hline 6 & $\begin{array}{l}\text { Number of US federal agencies, among } 24 \text { surveyed by the Government Accountability Office in 2021, } \\
\text { using facial recognition technology to generate leads in criminal investigations. }{ }^{8}\end{array}$ \\
\hline 10 & Agencies found in the same GAO study to be planning to expand its use in some manner. ${ }^{9}$ \\
\hline 13 & Billions of US dollars world facial recognition technology market is estimated to be worth by $2027 .{ }^{10}$ \\
\hline
\end{tabular}

ACM's Technology Policy Committees provide cutting-edge, apolitical, non-lobbying scientific information about all aspects of computing to policy makers in the United States and Europe. To tap the deep expertise of ACM's 100,000 members worldwide, contact ACM's Global Policy Office at acmpo@acm.org or +1 202.580.6555. 


\section{Facial recognition is not a single technology}

Facial recognition is not a monolithic technology or a particular technique. Rather, facial recognition refers to any technology that automatically processes and purports to identify faces in images or videos. While humans interpret faces easily, computers must extract patterns from data or humans must code patterns into the system. Applying these patterns yields the facial descriptors (often referred to as faceprints) on which facial recognition systems rely to achieve their function.

Facial recognition works by matching a probe image, such as a still from a surveillance camera or an eyewitness photograph (but not a sketch), to known images in a background set, like a database of identity card photos in which each image is clearly annotated with a specific identity. Whether employed for identification (assigning an identity) or verification (confirming a claimed identity), these systems seek to answer the question, "Does a background set image sufficiently match the probe image?"

Facial recognition systems usually rely on a quantitative similarity score of how alike two facial images are. Some systems also generate confidence scores, purporting to quantify how certain the system is about its claim of similarity. Such confidence scores, however, should be considered unreliable without extraordinary justification. ${ }^{11}$ Faceprints and scores are system specific and generally cannot be meaningfully compared between systems or interpreted directly.

\section{Privacy and facial recognition don't mix}

Today, anywhere there is a camera, individuals potentially can be identified and tracked. This affects the ability to move in public spaces without leaving identifiable traces.

\section{Corporations and governments have increasingly determined that the only responsible way to use facial recognition technology is to not use it at all.}

Opting out is rarely a meaningful option, as it implies that a person must never enter certain shared physical spaces with their face visible. Further, people in a public space cannot give meaningful consent without knowing how long surveillance data are retained or who might access them. Moreover, many commercial systems are developed using facial imagery gathered without the knowledge or consent of those depicted. Defenses, such as wearing a full-face mask, using substantial makeup, or averting one's face from cameras, are onerous and may be perceived by others as antisocial behavior. As a result, corporations and governments have increasingly determined that the only responsible way to use facial recognition technology is to not use it at all. ${ }^{12}$

\section{Facial recognition systems cannot be trusted in high-stakes applications}

Research has shown that facial recognition systems are not sufficiently accurate for applications in which

\section{Facial recognition systems are not sufficiently accurate for applications in which an individual's liberty may hang in the balance.}

an individual's liberty may hang in the balance or are otherwise high-stakes. Indeed, accuracy can vary drastically depending on the conditions under which the image is acquired (lighting, weather, pose, expression, concealment). High accuracy or low error are not, on their own, sufficient evidence of trustworthiness.

Moreover, valuing accuracy alone can be misleadingthe nature and distribution of failures matter. In many verification settings (say, unlocking a phone), even a very high number of false negatives (i.e., failures to match when a match should be found) might be tolerated, since it means only that the user must try again. But nearly any false positive (i.e., matching a face that should not be matched) could be disastrous, granting access when it should have been restricted.

While the developers and controllers of a facial recognition system can adjust the balance between false positives and false negatives, this simply trades one kind of error for another. Raising the threshold at which the system accepts similar faces as a match will reduce false positives. But by raising the threshold and reducing all matches, the system's usefulness may also be reduced. Beyond this, facial recognition systems cannot generalize beyond the faces on which they were developed. Showing a face from a person who is not part of the background set may, of necessity, cause the system to output an erroneous answer.

\section{Bias in facial recognition systems is pervasive and profound}

Research across many different systems and contexts 
has shown that errors and their sometimes severe consequences can fall disproportionately on minority populations. Facial recognition tools often perform less well for faces that do not resemble the majority of faces used in development (e.g., systems developed in Western countries perform poorly on Black and Asian faces, while ones developed in Asia perform poorly on Western and Black faces). Such deficiencies in data used to train the system are pervasive. Absent evidence

\section{Facial recognition systems should be presumed to perform poorly in assessing women and minority populations, particularly people of color.}

to the contrary, facial recognition systems should be presumed to perform poorly in assessing women and minority populations, particularly people of color. ${ }^{13}$ Other conditions also may contribute to bias, including physical changes due to illness or accident, hair loss, aging, and gender conversion.

A human "in the loop" does not automatically mitigate these issues. While humans are often involved in translating facial recognition outputs to real-world actions, the nature of these human-machine teams must be carefully examined to understand how successfully such systems function overall. For example, do scores or biases affect human judgments of identity, and if so, by how much?
The use of facial recognition for anything other than identification or verification is scientifically unsound

Many facial recognition systems also include features that, their developers claim, can reveal other information about an individual using facial analysis. Allegedly discernible characteristics include a subject's age, gender, race, emotional state, personality traits, sexual orientation, or even political affiliation. Although such systems have been deployed in highly sensitive applications from surveillance to education to hiring,

\section{There is no credible scientific evidence that any system built to identify a person can accurately and reliably determine anything else.}

there is presently no credible scientific evidence that any system built to identify a person is capable of accurately and reliably determining anything else. ${ }^{14}$ Indeed, these claims are of sufficiently questionable scientific validity that they recall the discredited discipline of phrenology, which purported to ascertain personality traits by measuring the shape and size of a person's skull. As a general rule, all facial recognition system performance claims should be replicable and subject to close scrutiny. Claims that such a system can make subjective judgments or reveal nonvisual information must be deemed suspect.

\section{KEY CONCLUSIONS}

Facial recognition represents a novel and pervasive risk to personal privacy and autonomy in public spaces.

High performance, including high accuracy, is not sufficient evidence of a facial recognition system's responsible use or trustworthiness.

All facial recognition systems should be presumed biased absent evidence to the contrary.

Claims that facial recognition systems can reliably determine any information or personal attribute other than identity are unlikely to withstand scientific scrutiny.

Responsible application of facial recognition technology requires careful, scientifically informed, and ongoing governance. 


\section{NOTES AND SOURCES}

1 https://www.freep.com/story/news/local/michigan/2021/04/13/detroit-police-wrongful-arrest-faulty-facialrecognition/7207135002/

2 http://extranet.greens-efa.eu/public/media/file/1/7297 as cited by https://www.euractiv.com/section/data-protection/ news/facialrecognition-technologies-already-used-in-11-eu-countries-and-counting-report-says/

3 Ibid.

4 https:/www.politico.eu/article/eu-data-protection-authorities-call-for-ban-on-facial-recognition/

5 Information compiled and presented by Fight for the Future at https://banfacialrecognition.com/map.

6 US population figures based on 2020 Census data officially reported at https:/www.census.gov/quickfacts/fact/table/ US/PST045221. Of this number, just under 60 million reside in New York and California, which have banned law enforcement use of facial recognition.

7 https://pages.nist.gov/frvt/reports/1N/frvt_1N_report.pdf

8 https://www.gao.gov/products/gao-21-526 and https://www.gao.gov/products/gao-21-518

9 Ibid.

10 https://www.fortunebusinessinsights.com/industry-reports/facial-recognition-market-101061

11 Estimating either confidence or prediction intervals for a function from facial imagery to individual identities is highly problematic. Although there is no generally accepted approach that works for all types of models, many approaches require knowledge or assumption of the distribution of facial features that is either unavailable or only estimated from the same data already used for system development, leaving unanswered the essential question of when the system's set of potential answers might be wrong.

12 Major tech companies such as Microsoft and Amazon have publicly pledged not to sell their facial recognition products to law enforcement. Meanwhile, governments at the city, county, state, and national level have either banned the procurement of this technology outright or for particular uses or periods of time. By February 2022, there were at least 20 cities, two counties, and two states that had banned the use of facial recognition by law enforcement; California and New York placed multiyear moratoria on such use. For an up-to-date list of proposed and effective legislation limiting facial recognition, consult Fight for the Future's map at https://banfacialrecognition.com/map.

13 For example, see Buolamwini, J. and Gebru, T. "Gender Shades: Intersectional accuracy disparities in commercial gender classification." In Proceedings of Machine Learning Research 81:1-15, 2018 Conference on Fairness, Accountability and Transparency. See also Grother, P., Ngan, M., and Hanaoka, K. NIST Face Recognition Vendor Test (FRVT), Part 3: Demographic Effects.

14 The models underlying facial recognition systems are often fundamentally correlation-based statistical models that lack a coherent scientific underpinning. See Malik, M. "A Hierarchy of Limitations in Machine Learning," https:// arxiv.org/abs/2002.05193. More specifically, see Agüera y Arcas, B., Mitchell, M., and Todorov, A. "Physiognomy's New Clothes,” Medium, posted May 6, 2017, https://medium.com/@blaisea/physiognomys-new-clothes-f2d4b59fdd6a.

\section{ADDITIONAL INFORMATION}

With 100,000 members in 190 countries, the nonprofit Association for Computing Machinery is the world's largest and longest-established organization of professionals involved in all aspects of computing. The association's global policy agenda concerning computing and information technology is guided by the ACM Technology Policy Council. Under the council's auspices, Technology Policy Committees in the United States and Europe provide cutting-edge, apolitical, non-lobbying information about computing and its social impacts to policy makers at all levels of government in many forms. These include public presentations, private briefings, public testimony, formal consultation, and rulemaking comments, as well as detailed reports and analyses.

\section{To tap the deep expertise of ACM's global membership, please contact ACM's Global Policy Office at acmpo@acm.org or +1 202.580.6555.}

\section{AUTHORSHIP \& ACKNOWLEDGEMENTS}

Dr. Joshua A. Kroll is an assistant professor of computer science at the Naval Postgraduate School (NPS) in Monterey, California. Views expressed are those of the author and do not reflect the position of NPS, the Department of Defense, or the U.S. government. This brief was produced for the ACM Technology Policy Council. It may be cited as "ACM Tech Brief: Facial Recognition," ACM Technology Policy Council (Issue 2, February 2022). 